\title{
Optimal Relation between ART and Mobility \& Transmission Range at Default QualNet \& Calculated Transmission Powers
}

\author{
Sachin Kumar Gupta ${ }^{1}$, Saeed Hamood Alsamhi ${ }^{2}$, and R. K. Saket ${ }^{3}$
}

\begin{abstract}
In Ad-hoc On-Demand Distance Vector (AODV) routing, it is suggested that the value of Active Route Timeout (ART) should be a constant (i.e. generalized for all kinds of applications or traffic generators). However, it has been observed from the current work that the choice of ART value according to network behavior and traffic generators may significantly increase the network performance. The presented work in this paper tries to analyze an optimal relation between the ART and node's mobility \& transmission ranges in which AODV network provides best performance. Here, AODV network performance is subjected to IEEE 802.11 MAC protocol under random waypoint topology. This paper uses the QualNet simulation tool to carry out the results, and the graphs have been prepared from obtained simulation results by using D-plot. Moreover, the Constant bitrate (CBR) traffic generator has been provided to generate the traffic between the mobile nodes and performance observation is based on throughput metric.
\end{abstract}

Keywords- AODV, ART, Node's Mobility \& Transmission Range, Default QualNet \& Calculated Transmission Power, CBR.

\section{INTRODUCTION}

$\mathrm{I}_{\mathrm{n}}^{\mathrm{N}}$ an infrastructure-less network, the mobile wireless network is commonly known as an ad hoc network $[1,2]$. It is a group of wireless mobile nodes creating a temporary network without the aid of any centralized administration [3]. Therefore, these kinds of networks are self-organizing, selfadministering and self-configuring multi-hop wireless networks, where the topology of the network changes dynamically due to node's mobility [4]. Due to the absence of infrastructure, the destination node might be out of the range of a source node for transmitting packets. Hence, an efficient routing procedure is always required to find a path between the source and the destination pairs for forwarding the packets.

Although, the number of ad hoc network routing protocols have been introduced in the market for various situations and applications. However, the main concern of this paper is AODV routing protocol.

Sachin Kumar Gupta is with the Department of Electronics and Communication Engineering, SMVD University, Katra-182320 (Jammu \& Kashmir) and Indian Institute of Technology (BHU), Varanasi-221005, (Uttar Pradesh,) India.

Saeed Hamood Alsamhi is with the Department of Electronics Engineering, IBB University, Ibb, Yemen and Indian Institute of Technology (BHU), Varanasi-221005, (Uttar Pradesh), India.

R. K. Saket is an Associate Professor with the Department of Electrical Engineering, Indian Institute of Technology (BHU), Varanasi-221005, (Uttar Pradesh,) India.
Like other reactive routing protocols, route discovery and route maintenance process is the key concept in AODV routing that deals with the topology changes [5]. Nevertheless, there also exist various factors, which influence the network performance more than the topology changes such as ART (i.e. holding time of route state information), network area, network load, node's mobility \& transmission range etc [6, 7]. The prime aim of this paper is to analyse how variation in ART value influences the performance of AODV routing protocol under different node's mobility and transmission range conditions at default QualNet transmission power and calculated transmission powers. Thereafter, this paper tries to point out an optimal relation between ART and node's mobility \& node's transmission range. Here, optimum relation among discussed factors is observed through throughput QoS metric. As per this paper, an optimal value of ART is the dynamic function of node's mobility \& transmission range. Hence, it could be said that the network performance may significantly increase by choosing a proper ART value according to network situations.

\section{ACTIVE_RouTE_TIMEOUT}

Although reactive protocols discover routes as and when required, they still maintain route state information for a short period in its cache to avoid the overhead of route establishment. This time duration when the state information is held is called Active_Route_Timeout (ART) [8]. In other words, ART is a period of time during which cached route is considered to be valid. When a cached route is not used for some period of time, the route state information of this cached route is removed by nodes from the routing table. The time, until the node removes the route state information about the cached route from the routing table, is called ART. After ART, cached route is considered to be expired, and its value can vary from protocol to protocol. When a route successfully establishes between two end points, it is remembered in case of its reuse in the future.

Basically, ART is a fixed parameter that tells how long route state information should be kept in the routing table after the last transmission of a packet from this route. The timer is reset back to ART whenever a route is used. The default value of this parameter is arbitrary set to 3 seconds in AODV routing, whereas it is known as Route Cache Timeout (RCT) in Dynamic Source Routing (DSR) routing and its default value is fixed at 300 seconds $[9,10]$. However, finding 
an optimal ART is not an easy task. It is a trade-off between opting a shorter ART resulting in a new route discovery process while the valid route is still there and a longer ART for sending the packets on an invalid route [11]. The first case introduces the delay in the network that could be avoided. The consequence of the second case is the loss of one or more packets and initiation of the Route Error (RERR) process instead of a new route discovery process.

\section{A. Impact of ART on Connectivity}

Consider Fig. 1, where a connection between node A and node $\mathrm{E}$ has been established through intermediate nodes $\mathrm{F}$ and $\mathrm{G}$ after the route discovery process. At the time of route discovery process, other routes have also been searched along the destination rather than the main communication link and these are $\mathrm{A}->\mathrm{B}->\mathrm{C}->\mathrm{D}->\mathrm{E}$ and $\mathrm{A}->\mathrm{I}->\mathrm{H}->\mathrm{C}->\mathrm{D}->\mathrm{E}$. These other routes along the destination are known as active routes, and they are valid up to 3 seconds by default. After 3 seconds, active routes will be invalid. Suppose, during communication one intermediate node $G$ is moved to $G$ ', then this session can be completed via other active routes without initializing the route discovery process.

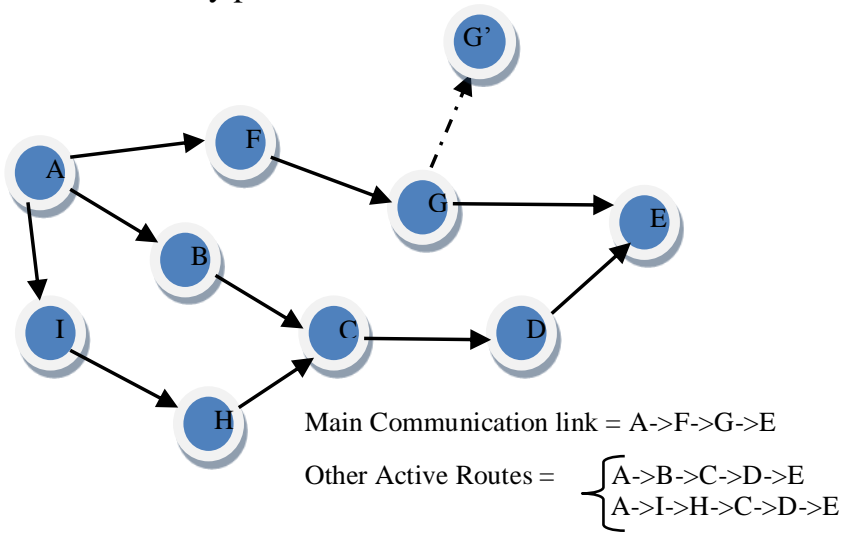

Fig. 1 Impact of ART on connectivity

\section{NODE's MoBILITY}

The node's mobility has a significant effect on the performance of AODV routing in an ad-hoc network, but how it will affect the performance of routing protocol is the matter of further examination. Fig. 2 depicts how the node's mobility affects the connectivity of the network. In case-1, Fig. 2 demonstrates the connection between nodes $\mathrm{X}$ and $\mathrm{Z}$ through node $\mathrm{Y}$. In other words, node $\mathrm{Y}$ plays the role of an intermediate node between these two parties where the node's mobility is low. However, in case-2, direct connection between these two parties is possible, only if the node $\mathrm{Z}$ will move to Z' position, and it is possible when node's mobility is high. On the other side, a very high value of node's mobility could influence the connectivity of the node, negatively [12, 13]. In other words, it could be said that an immediate change in the network topology may reduce the network performance because of higher node's mobility. In the case of lower node's mobility, node $\mathrm{Z}$ has to wait for a long time in order to get the direct link, or it may choose the intermediate/relay node to get the direct link than to wait.
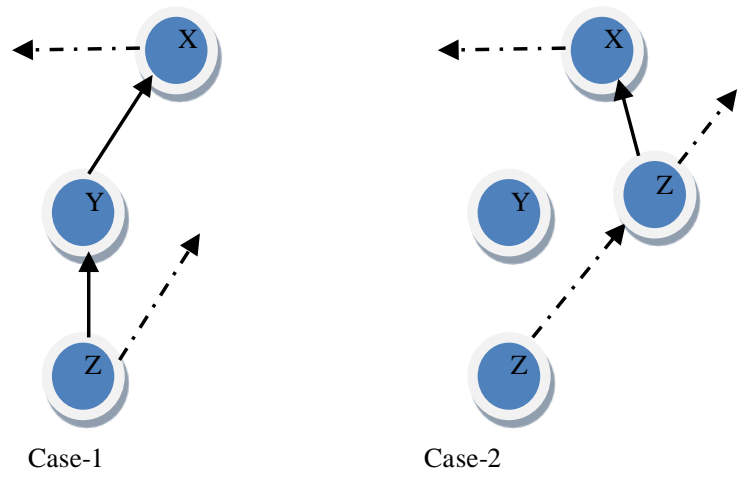

Fig. 2 Impact of node's mobility on connectivity

\section{NODE'S TRANSMISSION RANGE}

The energy conservation is a critical issue in an ad-hoc network environment because each node has limited battery power and it is not easy to replace/recharge the battery during communication $[14,15]$. Hence, it is important to utilize the battery power efficiently to ensure longer network lifetime by utilizing the optimum power for transmission [16].

As, it is the well known fact that the mobile nodes of an adhoc network are battery operated devices. Therefore, their transmission range is limited. If the transmission power is kept high then, although all the packets have been delivered, battery power consumption will get high. If it is kept less then, although the power consumption will be low, the packets may not be able to reach the destination. In order to maximize the battery life and to get better performance, an optimum value of transmission power is to be chosen for the respective transmission ranges.

\section{Simulation's Objective, Parameters \& OUtLine}

Although, the route discovery and route maintenance process are the key concept in AODV network environment that deal with the topology changes. However, various factors are there which influence network performance more than the topology changes. This study considers only a few factors, namely; mobility and transmission range of node along with the route maintenance parameters (here, ART is considered only). The main concern of this study is to find out the proper relation between ART and mobility \& transmission range at default QualNet transmission power and calculated transmission powers so that the network performance can be enhanced.

Already several researchers have attempted to observe the performance of AODV routing protocol by considering various factors like size of network, number of nodes in the given area, transmission range, mobility, movement pattern of nodes etc. However, they were restricted to only these factors and few of them separately considered the route maintenance parameters (like ART \& DPC only), but combine study between the route maintenance parameters and other various factors has not been studied so far. Therefore, this study presents the combined study between the route maintenance parameters (ART) and mobility \& transmission range in 
AODV network to observe the performance.

Here, QualNet simulation tool version ' 7.1 ' is used for creating and analyzing the simulation scenarios in order to study and observe the AODV routing performance under the influence of ART, mobility and transmission range at default QualNet and various calculated transmission powers which are obtained from equation (1).

$$
\begin{aligned}
& P(\text { watt })=\left[\frac{4 \pi X(\text { T.R. })}{0.1248575}\right]^{2} X 10^{-9.5} \\
& P(\mathrm{dbm})=10 X \log _{10}\left(P(\text { watt }) X 10^{3}\right)
\end{aligned}
$$

Where ' $\mathrm{P}$ ' is the transmission power in watt and ' $\mathrm{T}$. $\mathrm{R}$.' is the transmission range in meter.

Further, after creating the scenario, the CBR traffic generator has been introduced between the mobile nodes to generate the traffic with the rate of $2 \mathrm{KBps}$. Moreover, here each mobile node moves according to the random waypoint mobility model, where the value of pause time is 5 seconds. Here, the scenario simulation is run for the number of times for 300 seconds in order to get the best results, and their average value is chosen for plotting the graphs with the help of D-plot.

\section{A. Scenario Overview}

In this work, two different scenarios have been created in order to determine the proper combination of above discussed factors. These scenarios are as follows:

"Scenario-1: Throughput Vs ART for different Transmission Range; at default QualNet transmission power and calculated transmission powers"

In this section, throughput is shown as a function of ART for different values of transmission range at $15 \mathrm{dbm}$ default QualNet transmission power and various transmission powers of its respective transmission range that are calculated by equation (1). Here, 40 nodes (NLD=40) are spread randomly over an area of $1500 \mathrm{~m} \mathrm{X} 1500 \mathrm{~m}$ and they are moving according to the random waypoint mobility model. Among 40 nodes, eight node pairs have randomly been chosen as traffic generators. The main motive of this section is to analyze and to compare the impact of various transmission ranges on throughput. The simulation set-up of this scenario uses the following parameters that are listed in Table I.

TABLE I

SIMULATION PARAMETERS OF SCENARIO-1

\begin{tabular}{cc}
\hline ART & $0.5,1,3,5,7,9,11$ (in sec) \\
\hline Transmission Range & $\begin{array}{c}100,150,200,250,300,350,400, \\
450,500 \text { (in meter) }\end{array}$ \\
\hline $\begin{array}{c}\text { Transmission power for 11 Mbps } \\
\text { Network Load Density (NLD) }\end{array}$ & $\begin{array}{c}15 \mathrm{dBm} \& \text { Calculated Powers } \\
\text { Unicast connection between node or } \\
\text { offer loads in the network (SD pair) }\end{array}$ \\
\hline Node's Mobility & 5 (in mps) \\
\hline
\end{tabular}

"Scenario-2: Throughput Vs Node's Mobility for different Transmission Range; at default QualNet transmission power and calculated transmission powers, where ART=3 sec"

A scenario-2 has been considered to examine the throughput against the node's mobility for different values of transmission range at default ART value (i.e. ART=3). Moreover, this section observes the difference of throughput at default QualNet transmission power and calculated transmission powers. Again, the node placement and movement strategy are same here like scenario-1. Following parameters of Table II are considered while creating the simulation scenario-2.

TABLE II

SIMULATION PARAMETERS OF SCENARIO-2

\begin{tabular}{cc}
\hline Node's Mobility & $0.5,5,10,15,20$ (in mps) \\
\hline Transmission Range & $\begin{array}{c}200,250,300,350,400 \text { (in } \\
\text { meter) }\end{array}$ \\
Transmission power for 11 Mbps & $\begin{array}{c}15 \mathrm{dBm} \& \text { Calculated Powers } \\
\text { Network Load Density (NLD) }\end{array}$ \\
$\begin{array}{c}\text { Unicast connection between node } \\
\text { or offer loads in the network (SD } \\
\text { pair) }\end{array}$ & 80 \\
ART & 3 (in sec) \\
\hline
\end{tabular}

\section{B. $\quad$ Simulation Parameters}

Table III, IV and V tabulate the various other parameters that have also been chosen to create the both simulation scenarios at wireless subnet properties, node configuration, and scenario properties, respectively on the QualNet simulation tool.

TABLE III

WIRELESS SUBNET PROPERTIES

\begin{tabular}{cc}
\hline Physical Layer & \\
\hline Packet Reception Model & PHY802.11b \\
Channel Bandwidth & $11 \mathrm{Mbps}$ \\
Antenna Model & Omni directional \\
Antenna Height & $1.5 \mathrm{~m}$ \\
Antenna Efficiency & 0.8 \\
MAC Layer & \\
Mac Protocol & 802.11 \\
MAC Propagation Delay & $1 \mu \mathrm{s}$ \\
Network Layer & $\mathrm{IPv} 4$ \\
Network Protocol & \\
Routing Protocol & AODV \\
Routing Protocol IPv4 & 35 \\
Network Diameter & $40 \mathrm{~ms}$ \\
Node Traversal Time & 2 \\
Maximum_Route_Request_Retries & 100 \\
Maximum_Number_of_Buffer_Packets & 5 \\
Route Deletion Constant & Yes \\
Enable Hello Message & $1 \mathrm{~s}$ \\
Hello Interval & 2 \\
\hline Maximum Allowed Hello Loss & \\
\hline &
\end{tabular}


TABLE IV

NODE (DEVICE) CONFIGURATION

\begin{tabular}{|c|c|}
\hline Network Layer & $\mathrm{IPv} 4$ \\
\hline Routing Protocol & AODV \\
\hline Router Property & Generic \\
\hline \multicolumn{2}{|c|}{ Mobility and Placement } \\
\hline Mobility Model & Random waypoint \\
\hline Pause Time & $5 \mathrm{~s}$ \\
\hline \multicolumn{2}{|c|}{ Application Layer } \\
\hline Applications & CBR \\
\hline Packet Size & 512 Bytes/packet \\
\hline Packet Inter-Arrival Time & $0.25 \mathrm{~s}$ or 4 Packets/s \\
\hline Data Rate & $2 \mathrm{KBps}$ \\
\hline \multicolumn{2}{|c|}{$\begin{array}{c}\text { TABLE V } \\
\text { SCENARIO PROPERTIES }\end{array}$} \\
\hline \multicolumn{2}{|c|}{ General Parameters } \\
\hline Simulation Time & $300 \mathrm{~s}$ \\
\hline Terrain Size (Area) & $1500 \mathrm{~m} X 1500 \mathrm{~m}$ \\
\hline Number of Channels & 1 \\
\hline Channel Frequency & 2.4 GHz in Ad hoc mode \\
\hline Pathloss Model & Two ray \\
\hline Node Placement Strategy & Randomly \\
\hline
\end{tabular}

\section{RESUlTS DisCUSSION}

\section{A. Scenario-1}

Fig. 3 represents the result that has been obtained from this simulation scenario. This section tries to find out the impact of variation of transmission range on throughput at fixed transmission power. From Fig. 3, it can clearly be observed that initially the throughput increases as the node's transmission range increases. Further, as the transmission range is increased beyond 300 meters, the value of throughput starts to decline. Obviously, the default QualNet transmission power of $11 \mathrm{Mbps}$ channel bandwidth is not sufficient for higher transmission ranges such as 350, 400, 450 and 500 meters. At the outset until 300 meters of transmission range, throughput increases because the number of intermediate nodes or hops decreases with an increase in transmission range. It may be due to the degradation of the number of intermediate nodes or hops. Therefore, the possibility of route failure issues is also reduced and hence, throughput is increased. During 300 seconds of simulation, maximum unicast offered load is 16384 bps. However, at default QualNet transmission power, the maximum received throughput is only 13857.17 bps which is offered by a $300 \mathrm{~m}$ curve at ART $=1$. One can also notice from the Fig. 3 that at $\mathrm{ART}=1$, maximum throughput is acquired in the all cases of transmission range, whereas throughput values are almost constant for higher values of ART. It may be due to the constant node's mobility throughout the whole simulation. Hence, the changes in ART value will not affect the throughput.

Furthermore, the same situation is simulated at the different transmission powers (such as 15.00, 18.57, 21.07, 23.00, $24.59,25.93,27.00,28.10$, and $29.03 \mathrm{dbm}$ ) for its respective transmission range (like 100, 150, 200, 250, 300, 350, 400, 450 , and 500 meters respectively) instead of $15 \mathrm{dbm}$. Here, each node requires the different transmission power to obtain its respective transmission range that has been calculated from an equation (1) [17].

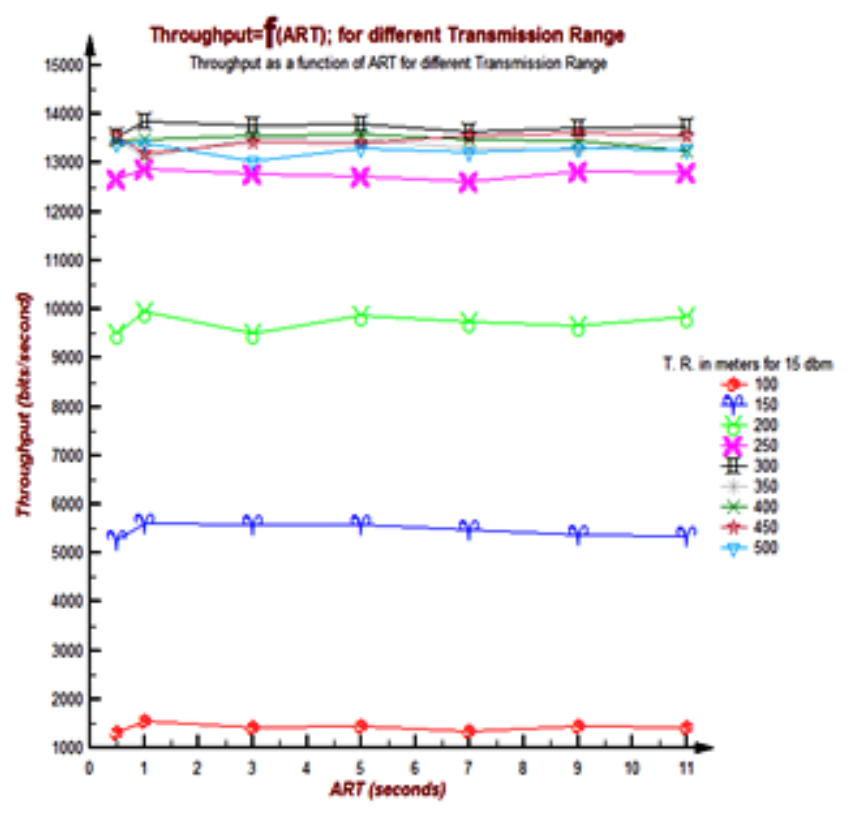

Fig. 3 Throughput Vs ART at default QualNet transmission power

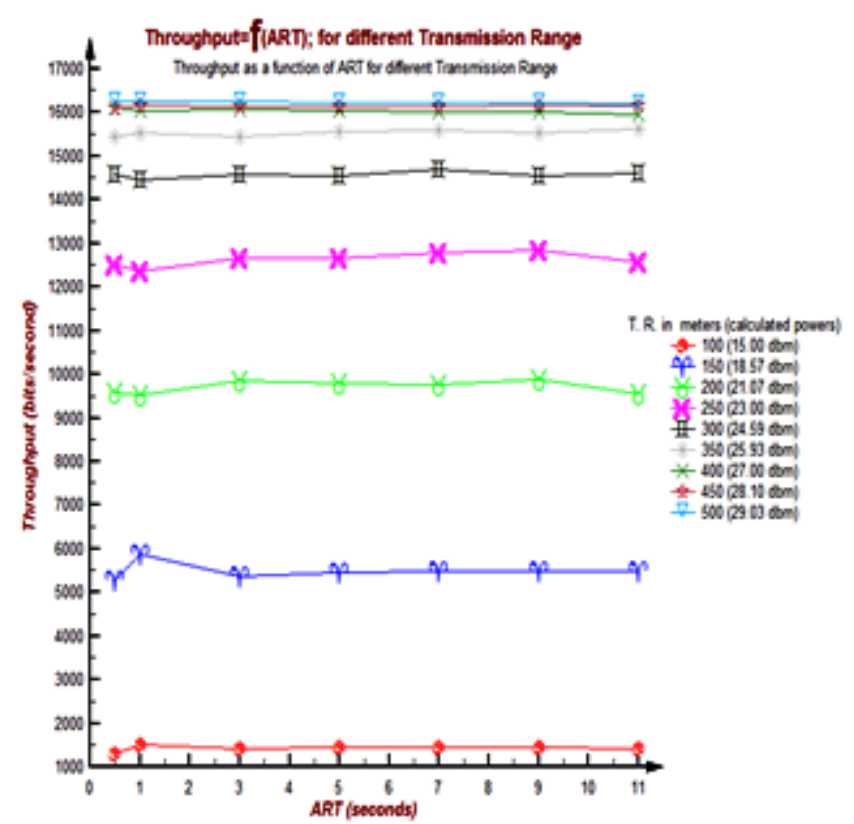

Fig. 4 Throughput Vs ART at calculated transmission powers

In this case, the value of throughput increases with an increase in transmission range from 100 to 350 meters. Later on, for the higher values of transmission range, it becomes almost constant as shown in Fig. 4. It is also noticeable that initially in lower ranges of transmission, the increasing ratio of throughput is high, and gradually this ratio decreases. Here, reason is same like Fig. 3. Finally, network acquires almost same throughput for higher ranges of transmission that is seen at 400,450 and 500 meters. It may be because, for these values of transmission range, the network has achieved its maximum throughput value. From the Fig. 4, one can also see that at higher values of transmission range throughput is nearly unchanged for all values of ART. This result is 
expected because there is no variation in node's mobility and hence changes in ART value, especially in higher transmission ranges, do not affect the throughput. In this case, during the whole simulation maximum received throughput is $16263.69 \mathrm{bps}$ out of $16384.00 \mathrm{bps}$. Table VI represents the comparison of average throughput/PDR for all taken transmission range at the default QualNet transmission power and calculated transmission powers.

TABLE VI

COMPARISON OF AVERAGE THROUGHPUT/PDR FOR ALL TAKEN TRANSMISSION RANGE AT DEFAULT QUALNET TRANSMISSION POWER AND CALCULATED TRANSMISSION POWERS

\begin{tabular}{|c|c|c|c|c|}
\hline $\begin{array}{c}\text { Transmission } \\
\text { Range } \\
\text { (meter) }\end{array}$ & \multicolumn{2}{|c|}{$\begin{array}{c}\text { Average Throughput \& } \\
\text { PDR at default QualNet } \\
\text { transmission power (bps) }\end{array}$} & \multicolumn{2}{|c|}{$\begin{array}{c}\text { Average Throughput \& } \\
\text { PDR at calculated } \\
\text { transmission powers (bps) }\end{array}$} \\
\cline { 2 - 5 } & Throughput & PDR & Throughput & PDR \\
\hline 100 & 1423.11 & 0.087 & 1428.83 & 0.087 \\
\hline 150 & 5455.04 & 0.332 & 5490.76 & 0.335 \\
\hline 200 & 9703.35 & 0.592 & 9751.75 & 0.595 \\
\hline 250 & 12759.02 & 0.779 & 12814.10 & 0.782 \\
\hline 300 & $\mathbf{1 3 7 2 4 . 8 4}$ (Max.) & $\mathbf{0 . 8 3 8}$ & 14574.36 & 0.889 \\
\hline 350 & 13583.89 & 0.829 & 15534.94 & 0.948 \\
\hline 400 & 13479.70 & 0.823 & 16031.18 & 0.978 \\
\hline 450 & 13473.09 & 0.822 & 16142.75 & 0.985 \\
\hline 500 & 13272.85 & 0.810 & $\mathbf{1 6 2 3 5 . 1 2}$ (Max.) & $\mathbf{0 . 9 9 0}$ \\
\hline
\end{tabular}

From the above comparison Table VI, it is clear that the default QualNet transmission power of 11 Mbps channel bandwidth is not sufficient for the higher values of transmission range like more than 300 . Hence, if higher values of transmission range are required, then it is suggested to use equation (1) to calculate the power for a respective transmission range.

\section{B. Scenario-2}

This sub-section shows and discusses the throughput graphs that have been plotted for different values of transmission range with node's mobility on the $\mathrm{X}$-axis and throughput on Y-axis.

Fig. 5 indicates the outcome that has been received from this simulation scenario at default QualNet transmission power (i.e. $15 \mathrm{dbm}$ ). From the Fig. 5 , it can be noticed that for lower ranges of transmission (i.e. $200 \& 250$ meters), the decreasing ratio of throughput with an increase in node's mobility is more than the higher ranges of transmission. Moreover, it can also be observed that for higher transmission ranges such as 300,350 and 400 meters, the throughput are almost constant especially at higher values of node's mobility. This result is expected because, at the higher ranges of transmission, the number of hops towards a particular destination is reduced. And hence, the possibilities of route breakage due to higher node's mobility are also minimized. Therefore, it may be concluded that the higher node's mobility, especially at higher values of transmission range does not affect the throughput as much. From the Fig. 5, it can also be noticed that the curve 300 acquires the maximum throughput. After that, performance starts to degrade because default transmission power is not sufficient for higher values of transmission range at this channel bandwidth.

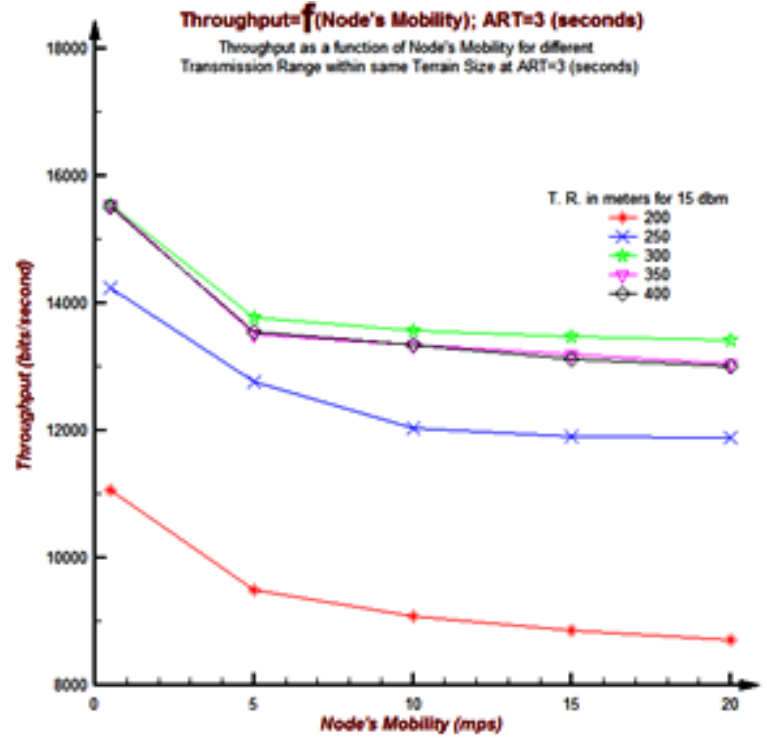

Fig. 5 Throughput Vs Node's Mobility at default transmission power

Again, the same case has been taken for analysis, but for various calculated transmission powers instead of its default power. Once more, the equation (1) is used for calculating the various transmission powers to achieve respective transmission range by each node. From the Fig. 6, it can be observed that the $400 \mathrm{~m}$ curve delivers the highest throughput. Now, in this case, nodes can achieve the 400 meters of coverage distance because of $27.00 \mathrm{dbm}$ transmission power. Again, the network performance decreases here with an increase in node's mobility. Moreover, at the higher range of transmission like 400 meters, the network performance is nearly unchanged for all node's mobility. The reason is same as in the previous case.

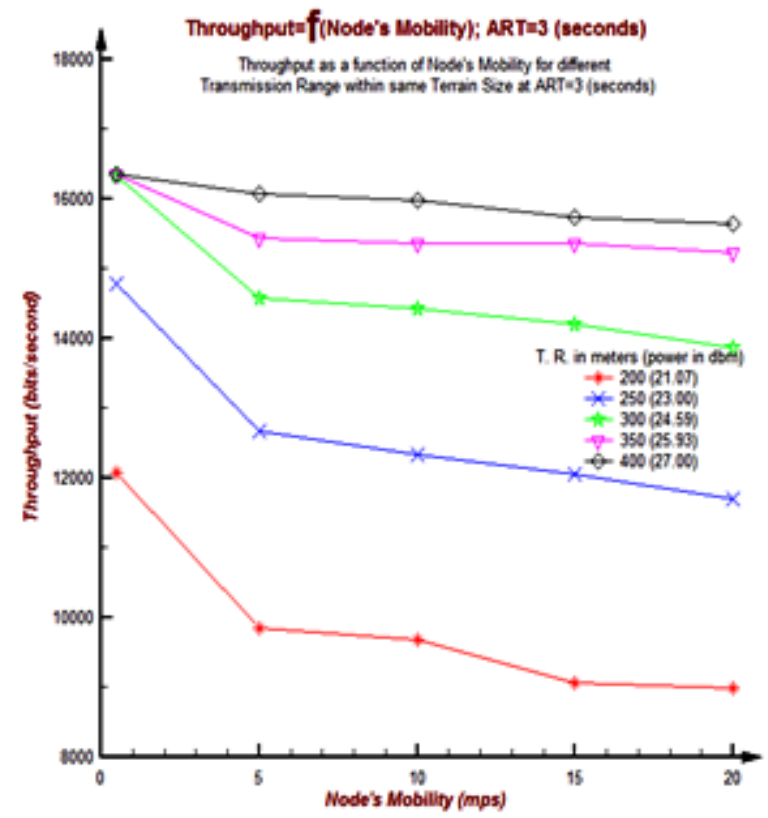

Fig. 6 Throughput Vs Node's Mobility at calculated transmission powers 


\section{CONCLUSION}

The main motive to consider this study is to analyze the effect of various transmission ranges on throughput at default QualNet transmission power and calculated transmission powers. The first scenario concludes that throughput value increases up to 300 meters of the transmission range. After 300 meters, the value of throughput is decreased, as the channel bandwidth has been fixed at $11 \mathrm{Mbps}$. Therefore, the $15 \mathrm{dbm}$ transmission power is not adequate on this channel bandwidth to transmit the packets beyond 300 meters. Till 300 meters, the number of hops reduces as the transmission range increases, which in turn reduces the possibility of route breakage. Here, it can also be noticed that at ART $=1$, maximum throughput is acquired almost for all transmission ranges, and it is almost constant for other higher ART values. It may be due to the constant node's mobility throughout the simulation (here, it is fixed at $5 \mathrm{mps}$ ). Hence, changes in ART value do not affect the throughput. Moreover, the same condition has been analyzed for the calculated transmission powers. In this case, the value of throughput increases up to 350 meters of transmission range. Later on, the throughput becomes almost constant for higher values of transmission range. In second scenario, for all transmission ranges, the value of throughput is higher at lower node's mobility. And, it is almost constant at higher values of node's mobility which is seen especially in higher transmission ranges. The main reason for this outcome is reduction in the number of hops towards a particular destination as transmission range increases. Hence, higher values of node's mobility do not have much impact on throughput.

\section{REFERENCES}

[1]. M. P. Johansson, and P. Larsson, "Wireless ad hoc networking: the art of networking without a network", Ericsson Rev. 4, pp. 248-263, 2000.

[2]. IETF Working Group, "Mobile Ad hoc Networks (MANET)", http://www.ietf.org/html. charters/manet-charter.html

[3]. D. B. Johnson, and D. A. Maltz, "Dynamic source routing in ad hoc wireless networks", In: Imielinski, T., Korth, H. (eds.) Mobile Computing, Chapter 5, Kluwer Academic Publishers, Berlin, pp. 153$181,1996$. https://doi.org/10.1007/978-0-585-29603-6_5

[4]. X. Hong, X. Kaixin, and M. Gerla, "Scalable routing protocols for mobile ad hoc networks", IEEE Netw. Mag. Global Internetw, vol. 16, no. 4, pp. 11-21, 2002. https://doi.org/10.1109/MNET.2002.1020231

[5]. S. K. Gupta, and R. K. Saket, "Performance metric comparison of AODV and DSDV routing protocols in MANETs using NS-2", International Journal of Research and Reviews in Applied Sciences, vol. 7, no. 3, pp. 339-350, 2011.

[6]. Y. Chen, and W. Wang, "The measurement and auto-configuration of ad-hoc", 14th IEEE 2003 International Symposium on Personal, Indoor and Mobile Radio Communication, vol. 2, pp. 1649-1653, Sep. 2003.

[7]. S. K. Gupta, and R. K. Saket, "Effect of ART, DPC and ANs on the Performance of AODV Routing," IEEE 10th International Conference on Industrial and Information Systems (ICIIS), Faculty of Engineering, University of Peradeniya, Sri Lanka, pp. 77-81, Dec 2015. https://doi.org/10.1109/iciinfs.2015.7398989

[8]. C. Perkins, "Ad-Hoc On-Demand Distance Vector (AODV) Routing", Internet-Draft, draft-ietf-manet-aodv-00.txt, Nov 1997.

[9]. C. Richard, C.E. Perkins, and C. Westphal, "Defining an Optimal Active Route Timeout for the AODV Routing Protocol", 2nd Annual IEEE Communications Society Conference on Sensor and $\mathrm{Ad} \mathrm{Hoc}$ Communications and Networks, IEEE SECON Poster Session, Nokia Research Center, Santa Clara, California, USA, pp. 1-3, 2005.
[10]. S. K. Gupta, R. Sharma, and R. K. Saket, "Effect of variation in active route timeout and delete period constant on the performance of AODV protocol”, International Journal of Mobile Communications (IlMC), vol. 12, no. 2, pp. 177-191, 2014. https://doi.org/10.1504/IJMC.2014.059737

[11]. S. K. Gupta, and R. K. Saket, "Observation of AODV Routing Protocol's Performance at Variation in ART Value for Various Node's Mobility", Smart Innovation, Systems and Technologies, Springer International Publishing Switzerland, vol. 50, pp. 419-427, 2016, DOI 10.1007/978-3-319-30933-0_42. https://doi.org/10.1007/978-3-319-30933-0_42

[12]. Y. Chen, and W. Wang, "The Measurement and Auto-Configuration of Ad-hoc", IEEE 14th International Symposium on Personal, Indoor and Mobile Radio Communications, vol. 2, pp. 1649-1653, Sep 2003.

[13]. K. Amjad, and A. J. Stocker, "Impact of node density and mobility on performance of AODV and DSR in MANET", 7th International Symposium on Communication Systems Networks and Digital Signal Processing, Newcastle upon Tyne, United Kingdom, pp. 61-65, Jul 2010.

[14]. J. Tripathi, J. C. de Oliveira, and J. P. Vasseur, "Proactive versus reactive routing in low power and lossy networks: Performance analysis and scalability improvements", Ad Hoc Networks, vol 23, pp. 121-144, Dec 2014. https://doi.org/10.1016/j.adhoc.2014.06.007

[15]. J. Tripathi, J. de Oliveira, and J. P. Vasseur, "Performance Evaluation of the Routing Protocol for Low-Power and Lossy Networks (RPL)", RFC: 6687, Oct 2012. http://tools.ietf.org/html/rfc6687.

[16]. P. Levis, A. Tavakoli, and S. Dawson-Haggerty, "Overview of Existing Routing Protocols for Low Power and Lossy Networks", Internet-Draft, Apr 2009. http://tools.ietf.org/html/draft-ietf-roll-protocols-survey-07.

[17]. W. Al-Mandhari, K. Gyoda, and N. Nakajima, "Performance Evaluation of Active Route Time-Out parameter in Ad-hoc On Demand Distance Vector (AODV)", 6th WSEAS International Conference on Applied Electromagnetic, Wireless and Optical Communications, Trondheim, Norway, pp. 47-51, Jul 2008. 